\title{
The Effect of the Oral PKC B Inhibitor Ruboxistaurin on Vision Loss in Two Phase 3 Studies
}

\section{Citation}

Sheetz, Matthew J., Lloyd Paul Aiello, Matthew D. Davis, Ronald Danis, Toke Bek, Jose CunhaVaz, Nazila Shahri, and Paul H. Berg. 2013. The Effect of the Oral PKC B Inhibitor Ruboxistaurin on Vision Loss in Two Phase 3 Studies. Invest. Ophthalmol. Vis. Sci. 54, no. 3: 1750. doi:10.1167/ iovs.12-11055.

\section{Published Version}

doi:10.1167/iovs.12-11055

\section{Permanent link}

http://nrs.harvard.edu/urn-3:HUL.InstRepos:35773005

\section{Terms of Use}

This article was downloaded from Harvard University's DASH repository, and is made available under the terms and conditions applicable to Other Posted Material, as set forth at http:// nrs.harvard.edu/urn-3:HUL.InstRepos:dash.current.terms-of-use\#LAA

\section{Share Your Story}

The Harvard community has made this article openly available.

Please share how this access benefits you. Submit a story.

\section{Accessibility}




\title{
The Effect of the Oral PKC $\beta$ Inhibitor Ruboxistaurin on Vision Loss in Two Phase 3 Studies
}

\author{
Matthew J. Sheetz, ${ }^{1}$ Lloyd Paul Aiello, ${ }^{2}$ Matthew D. Davis, ${ }^{3}$ Ronald Danis, ${ }^{3}$ Toke Bek, ${ }^{4}$ \\ Jose Cunha-Vaz, ${ }^{5}$ Nazila Shabri, ${ }^{6}$ and Paul H. Berg, ${ }^{1}$ for the MBDL and MBCU Study Groups ${ }^{7}$
}

Purpose. To assess the effect of ruboxistaurin (RBX) on vision loss through a prospectively defined combined analysis of two phase 3 trials (MBDL and MBCU).

Methods. Patients in both of these 3-year randomized, placebo-controlled, double-masked trials had best-corrected Early Treatment Diabetic Retinopathy Study (ETDRS) visual acuity (VA) $\geq 75$ letters ( 20/32 Snellen), ETDRS retinopathy level 20 to 47D (MBDL) or 35B to 53E (MBCU), and no prior panretinal or focal photocoagulation in at least one eye at baseline. Patients received oral placebo $(N=508$ total from both studies) or RBX $32 \mathrm{mg} / \mathrm{d}(N=520$ total). Best-corrected ETDRS VA was measured at 6-month intervals for 3 years (MBDL) or for 18 to 48 months (MBCU). Sustained moderate visual loss (SMVL) was defined as a 15-letter or more reduction from baseline in VA sustained for a patient's last 6 months of study participation.

Results. In the combined studies ( $N=1028$ total), SMVL occurred in $4.4 \%$ of placebo- versus $2.3 \%$ of RBX-treated patients $(P=0.069)$. In patients with a minimum of 2 years of follow-up ( $N=825$ total), there was less SMVL in the RBX group ( $4.4 \%$ placebo versus $2.1 \% \mathrm{RBX}, P=0.045$ ). Other VArelated measures (mean VA, contrast sensitivity, Visual Functioning Questionnaire 25 [VFQ-25]) either trended toward a benefit for RBX or were also statistically significant in favor of RBX. In contrast, diabetic macular edema (DME) morphologyrelated measures (occurrence of significant center of macula involvement, optical coherence tomography [OCT]-determined center of macula thickness, application of focal photocoagulation) did not show a consistent trend in favor of or against RBX.

From ${ }^{1}$ Eli Lilly and Company, Indianapolis, Indiana; the ${ }^{2}$ Joslin Diabetes Center, Beetham Eye Institute, Department of Ophthalmology, Harvard University Medical School, Boston, Massachusetts; the ${ }^{3}$ Department of Ophthalmology and Visual Sciences, University of Wisconsin-Madison, Madison, Wisconsin; the ${ }^{4}$ Department of Ophthalmology, Aarhus University Hospital, Aarhus, Denmark; the ${ }^{5}$ Association for Innovation and Biomedical Research on Light and Image (AIBILI) and the Faculty of Medicine, University of Coimbra, Coimbra, Portugal; and ${ }^{6} \mathrm{i} 3$ Corporation, San Diego, California.

${ }^{7}$ See the Appendix for the members of the MBDL and MBCU Study Groups.

Supported by Eli Lilly and Company, Indianapolis, Indiana.

Submitted for publication September 28, 2012; revised December 17, 2012 and January 24, 2013; accepted January 31, 2013.

Disclosure: M.J. Sheetz, Eli Lilly and Company (I, E); L.P. Aiello, Eli Lilly and Company (C); M.D. Davis, Eli Lilly and Company (C); R. Danis, Eli Lilly and Company (C); T. Bek, None; J. Cunha-Vaz, None; N. Shahri, Eli Lilly and Company (C); P.H. Berg, Eli Lilly and Company (I, E)

Corresponding author: Matthew J. Sheetz, Lilly Corporate Center, Indianapolis, IN 46285; msheetz@lilly.com.
Conclusions. SMVL data in a prospectively defined combined analysis from these two phase 3 trials suggest a magnitude of effect of RBX on vision loss similar to that seen in two prior studies (approximately 50\% reduction above standard care). However, event rates were low and statistical significance was not achieved. (ClinicalTrials.gov numbers, NCT00133952, NCT00090519.) (Invest Ophthalmol Vis Sci. 2013;54:17501757) DOI:10.1167/iovs.12-11055

T $\mathrm{n}$ the United States, diabetic retinopathy (DR) is the leading 1 cause of new-onset blindness among adults aged 20 to 74 years. ${ }^{1}$ The primary cause of vision loss in patients with DR is diabetic macular edema (DME) involving the center of the macula. ${ }^{2}$ DME is a complication of DR characterized by retinal thickening that is thought to arise principally from leaking retinal capillaries. The molecular mechanisms by which diabetes leads to DR (and subsequently to DME) include possible roles for hyperglycemia-induced oxidative stress, polyol pathway activation, production of advanced glycation end products, and activation of the diacylglycerol-protein kinase $\mathrm{C}(\mathrm{PKC})$ pathway. ${ }^{3-5}$

Ruboxistaurin (RBX), an orally administered isoform-selective inhibitor of PKC $\beta$, has been shown to have a beneficial effect in animal models of DR, ${ }^{6-9}$ and has also been shown to ameliorate diabetes-induced retinal hemodynamic abnormalities in patients with diabetes. ${ }^{10}$

Two prior 3-year randomized, double-masked, placebocontrolled phase 3 trials (PKC-DRS and PKC-DRS2) ${ }^{11,12}$ demonstrated an approximately $50 \%$ reduction in the occurrence of vision loss in patients treated with $\mathrm{RBX}$, although one of the trials (PKC-DRS) was not adequately powered to achieve statistical significance. ${ }^{11}$

Here we report the results of two additional randomized, double-masked, placebo-controlled phase 3 trials of RBX in patients with DME. Study MBDL was a 3-year trial in 731 patients with DME that was, in at least one eye, less severe than that required to meet the definition of clinically significant macular edema (CSME, see Table 1). Study MBCU was an 18- to 48 -month trial in 309 patients who had, in at least one eye, CSME that did not involve or imminently threaten the center of the macula. The initial aim of each trial was to investigate the effect of RBX on DME progression (as described in "Patients and Methods" below); but, following a request by the U.S. Food and Drug Administration for additional vision loss efficacy data, the protocols and/or statistical analysis plans were amended to make the primary aim the investigation of the effect of RBX on vision loss. In addition, both protocols were amended to indicate that vision loss data were to be combined across the two studies to maximize statistical power, given that neither study was originally designed to test the effect of $\mathrm{RBX}$ on vision loss. 
TABLE 1. Key Eye-Related Inclusion Criteria

MBDL MBCU

\begin{tabular}{lll}
\hline DME study eye & & $\geq 75$ \\
Visual acuity (letters correct) & 75 & CSME, but $>100 \mu \mathrm{m}$ from COM \\
DME severity & $>500 \mu \mathrm{m}$ from COM, no CSME & $35 \mathrm{~B}$ to 53E \\
DR severity (ETDRS level) & 20 to 47D & None for DR or DME \\
Prior PC & None for DR or DME & \\
Fellow eye* & & Any \\
Visual acuity (letters correct) & Any & Any \\
DME severity & Any & $<$ PDR \\
DR severity & $<$ PDR & No PRP \\
Prior PC & No PRP & \\
\hline
\end{tabular}

COM, center of macula; CSME, defined as DME within $500 \mu \mathrm{m}$ of the center of the macula or DME $>1$ disc area in size, part of which is within $1500 \mu \mathrm{m}$ of the center of the macula; PC, photocoagulation.

* Criteria for inclusion as a DR study eye.

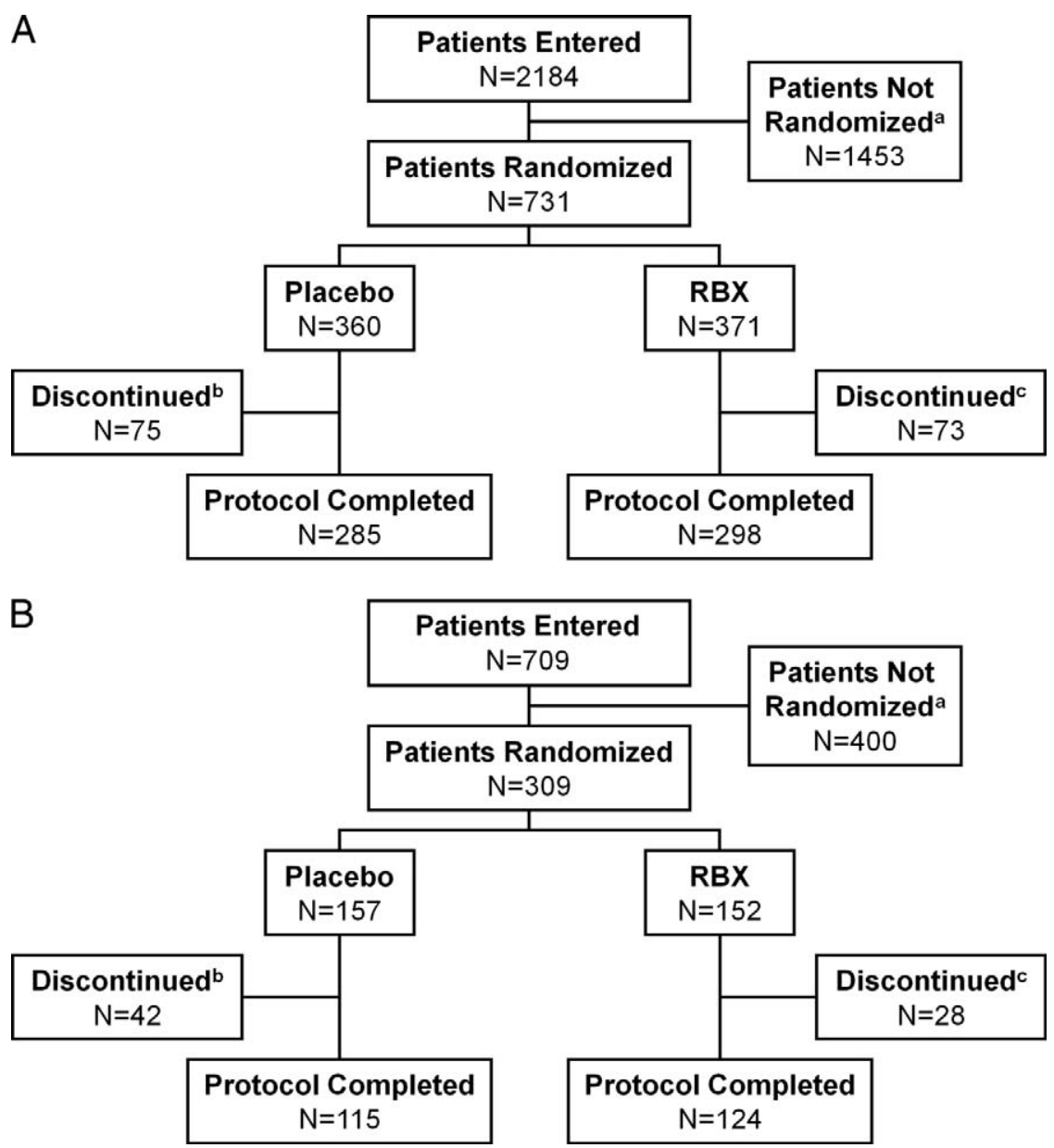

Figure 1. Patient disposition in (A) Study MBDL and (B) Study MBCU. MBDL footnotes (A): ${ }^{2}$ Reasons not randomized: protocol entry criteria not met (1192 patients), patient decision (52 patients), physician decision (22 patients), sponsor decision ( 2 patients), unable to contact patient (8

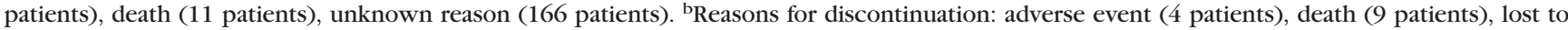
follow-up (29 patients), patient decision ( 29 patients), physician decision ( 3 patients), sponsor decision ( 1 patient). ${ }^{\mathrm{C}}$ Reasons for discontinuation: adverse event ( 5 patients), death ( 9 patients), lost to follow-up (26 patients), patient decision ( 28 patients), physician decision ( 2 patients), sponsor decision (3 patients). MBCU footnotes (B): ${ }^{2}$ Reasons not randomized: protocol entry criteria not met (379 patients), patient decision (14 patients), physician decision ( 2 patients), sponsor decision ( 1 patient), unable to contact patient (3 patients), unknown reason ( 7 patients). ${ }^{b}$ Reasons for discontinuation: adverse event ( 1 patient), death ( 3 patients), lost to follow-up ( 9 patients), patient decision ( 20 patients), physician decision ( 4 patients), protocol entry criteria not met (4 patients), protocol violation (1 patient). ${ }^{c}$ Reasons for discontinuation: adverse event ( 2 patients), death ( 2 patients), lost to follow-up (14 patients), patient decision (4 patients), physician decision (3 patients), protocol entry criteria not met (1 patient), protocol violation (1 patient), sponsor decision (1 patient). 
TABLE 2. Patient Characteristics at Baseline

\begin{tabular}{|c|c|c|c|c|}
\hline \multirow[b]{2}{*}{ Treatment, No. of Patients } & \multicolumn{2}{|c|}{ MBDL } & \multicolumn{2}{|c|}{ MBCU } \\
\hline & PBO, $N=360$ & $\mathbf{R B X}, N=371$ & PBO, $N=157$ & $\mathbf{R B X}, N=152$ \\
\hline Age, $y$, mean $\pm S D$ & $55 \pm 11$ & $55 \pm 11$ & $56 \pm 9$ & $55 \pm 10$ \\
\hline Sex, $\%$ male & 62 & 63 & 58 & 57 \\
\hline Origin, \% Caucasian & 74 & 77 & 75 & 74 \\
\hline Diabetes type, \% type 2 & 79 & 77 & 89 & 83 \\
\hline Diabetes duration, $y$, mean $\pm \mathrm{SD}$ & $16 \pm 7$ & $16 \pm 8$ & $15 \pm 9$ & $15 \pm 8$ \\
\hline Insulin use, $\%$ & 63 & 65 & 55 & 63 \\
\hline HbA1c, $\%$, mean $\pm \mathrm{SD}$ & $8.3 \pm 1.3$ & $8.1 \pm 1.3$ & $8.2 \pm 1.3$ & $8.1 \pm 1.3$ \\
\hline BMI, $\mathrm{kg} / \mathrm{m}^{2}$, mean $\pm \mathrm{SD}$ & $30 \pm 6$ & $30 \pm 6$ & $30 \pm 5$ & $31 \pm 6$ \\
\hline
\end{tabular}

$\mathrm{PBO}$, placebo; RBX, ruboxistaurin $32 \mathrm{mg} / \mathrm{d}$.

\section{Patients and Methods}

For both studies, entry criteria included a diagnosis of type 1 or 2 diabetes, age $\geq 18$ years, hemoglobin A1c (HbA1c) $\leq 11 \%$, and blood pressure $<160 / 90$ (MBCU) or mean arterial pressure $\leq 113$ (MBDL). Key eye-related inclusion criteria are summarized in Table 1 . For the purposes of assessing visual acuity, vision loss, and DR progression in both studies, if the fellow eye had proliferative diabetic retinopathy (PDR) or a history of panretinal photocoagulation (PRP) at baseline, then only the eligible DME study eye was considered a "DR" study eye. Otherwise, both eyes were considered DR study eyes (even if the fellow eye had been previously treated with focal photocoagulation for DME). These studies were originally intended to show an effect of RBX on morphologic progression of DME using grading of Early Treatment Diabetic Retinopathy Study (ETDRS) stereo fundus photographs to determine center of macula involvement (for Study MBDL) and optical coherence tomography (OCT) assessment of central macular thickness (for Study MBCU), with one designated DME study eye of each patient being used for the primary analyses. However, when a regulatory need for more vision loss data with RBX arose, the studies were amended to make vision loss in one or two DR study eyes the primary objective, consistent with prior studies of the effects of RBX on vision loss. ${ }^{11,12}$ In each study, more than $90 \%$ of patients had two DR study eyes.

With regard to the treatment of DME or DR during the study, patients were allowed to receive focal photocoagulation (FPC) and/or PRP in either eye postbaseline, at the discretion of the investigator. Application of FPC was captured on a specific case report form. Both trials started before intravitreal injections of steroids or anti-VEGF agents were approved for the treatment of DME, and application of these treatments was not captured on a specific case report form. However, their use was not prohibited in either study.

Both trials were multicenter, parallel, placebo-controlled, doublemasked, phase 3 clinical trials. Patients in the MBDL study were randomized to placebo $(N=360)$ or $32 \mathrm{mg} / \mathrm{d}$ oral $\mathrm{RBX}(N=371)$ and were followed for 36 months. Recruitment began in March 2004, and the last follow-up visit was in April 2010. Patients in the MBCU study were randomly assigned to receive oral placebo $(N=157)$ or RBX 32 $\mathrm{mg} / \mathrm{d}(N=152)$ and were followed for 18 to 48 months. The reason for the wide range of follow-up durations in the MBCU study is that it was originally designed as an 18-month DME progression study but was amended to incorporate a longer follow-up duration in order to better assess effects of RBX on vision loss. Prior to the amendment, approximately one-third of patients had already completed the final (18 month) study visit of the original protocol. Following the amendment, the minimum duration of follow-up was 24 months, and all active patients remained in the study until the final patient randomized reached 24 months of follow-up. Recruitment began in August 2005, and the last follow-up visit was in July 2011.

Best-corrected ETDRS visual acuity (VA) was measured at baseline and at 6-month intervals throughout both studies. The endpoint of sustained moderate visual loss (SMVL) has been described previously ${ }^{13}$ and was defined as a 15-letter or more decrease from baseline in VA that is sustained for a patient's last 6 months of study participation. DR severity was determined through central grading of ETDRS 7-standard field stereoscopic photographs and was assessed at baseline and at

TABLE 3. Eye Characteristics at Baseline

\begin{tabular}{|c|c|c|c|c|}
\hline \multirow[b]{2}{*}{ Treatment, No. of Study Eyes } & \multicolumn{2}{|c|}{ MBDL } & \multicolumn{2}{|c|}{ MBCU } \\
\hline & PBO, $N=696$ & $\mathbf{R B X}, N=722$ & PBO, $N=304$ & $\mathbf{R B X}, N=292$ \\
\hline ETDRS VA, letters correct, mean \pm SD (Snellen equivalent) & $84 \pm 8(20 / 20)$ & $84 \pm 8(20 / 20)$ & $84 \pm 6(20 / 20)$ & $83 \pm 8(20 / 25)$ \\
\hline \multicolumn{5}{|l|}{ DME, \% of DR study eyes } \\
\hline None to $<1 / 6 \mathrm{DA}$ & 19 & 19 & 17 & 16 \\
\hline$\geq 1 / 6$ DA to $>500 \mu \mathrm{m}$ from $\mathrm{COM}$ & 62 & 61 & 10 & 9 \\
\hline CSME $>100 \mu \mathrm{m}$ from COM & 12 & 10 & 43 & 45 \\
\hline CSME $<100 \mu \mathrm{m}$ from COM or questionably involving COM & 3 & 3 & 19 & 17 \\
\hline CSME definitely involving COM & 4 & 4 & 10 & 14 \\
\hline \multicolumn{5}{|l|}{ DR, \% of DR study eyes } \\
\hline Mild to moderate NPDR (ETDRS 20-43) & 61 & 64 & 52 & 50 \\
\hline Moderately severe NPDR (ETDRS 47) & 39 & 35 & 42 & 47 \\
\hline Severe NPDR (ETDRS 53) & $<1$ & 1 & 6 & 3 \\
\hline OCT center point thickness, $\mu \mathrm{m}$, mean $\pm \mathrm{SD}$ & ND & ND & $189 \pm 50$ & $191 \pm 64$ \\
\hline OCT central subfield thickness, $\mu \mathrm{m}$, mean $\pm \mathrm{SD}$ & ND & ND & $213 \pm 33$ & $214 \pm 31$ \\
\hline
\end{tabular}

ND, not determined. 
A

\section{Overall}

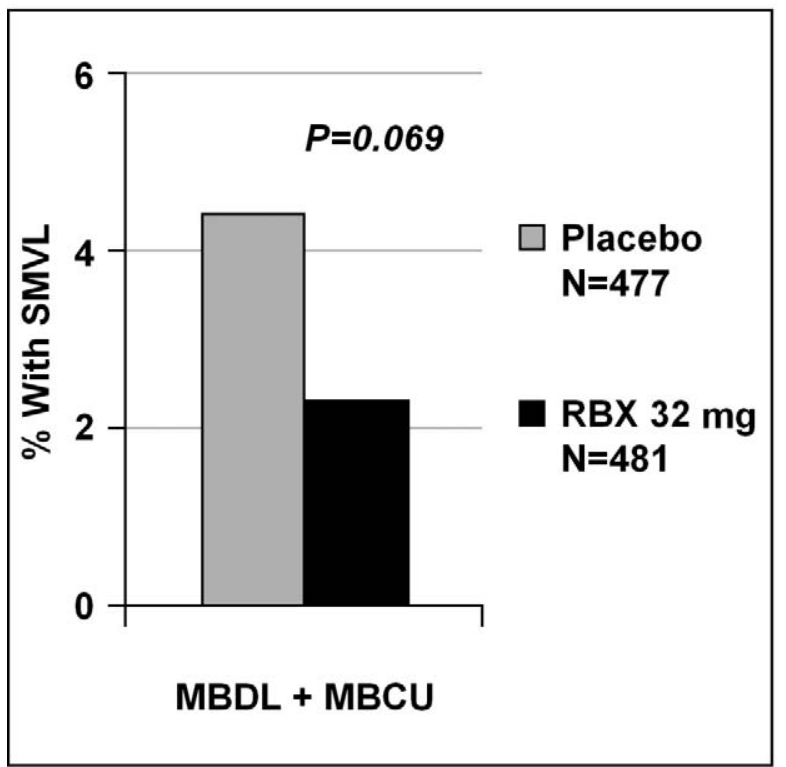

\section{Yr Min}

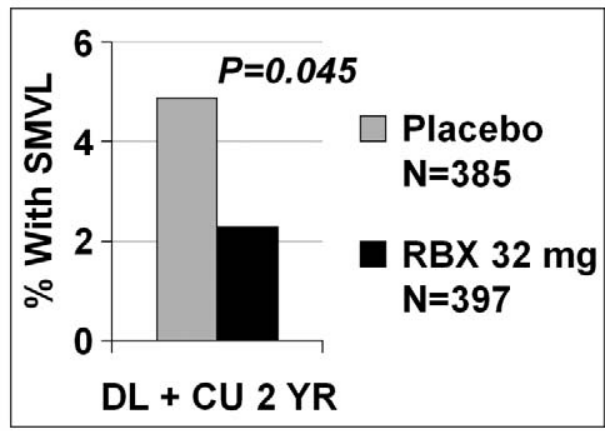

3 Yr Min

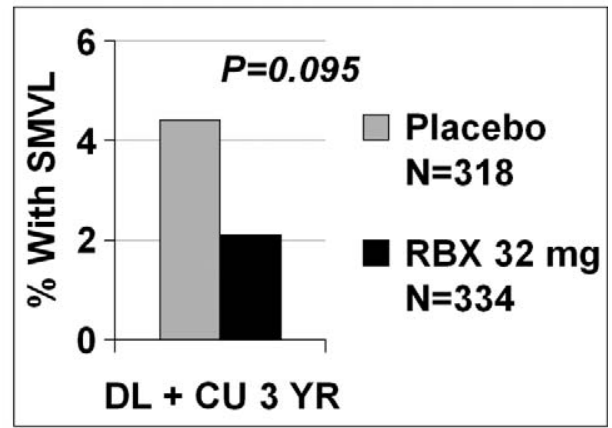

B
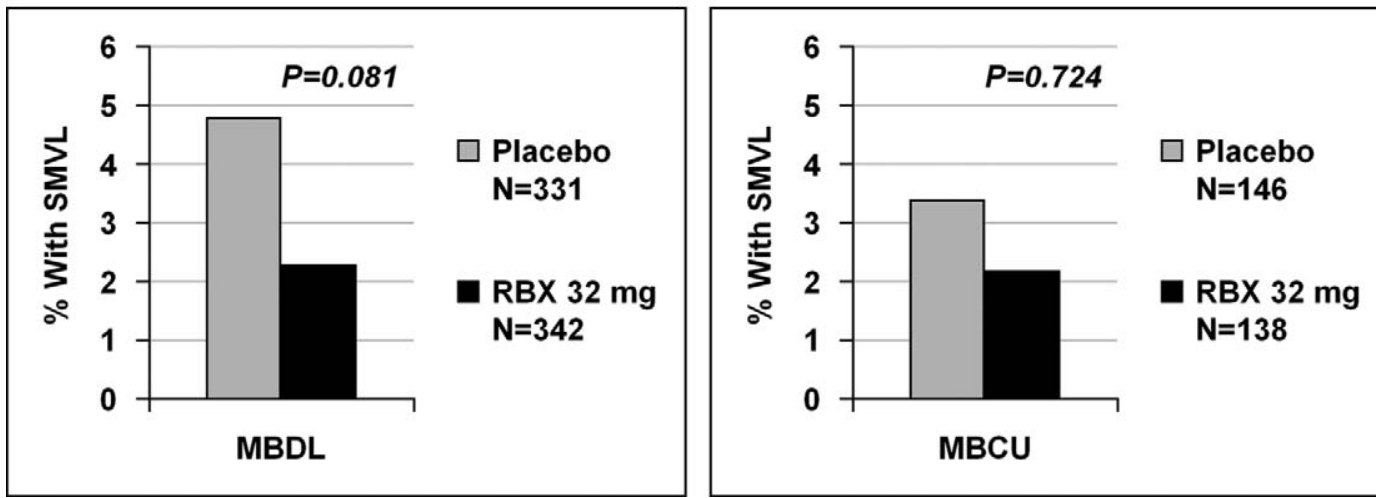

Figure 2. Primary outcome-sustained moderate visual loss (SMVL). Percent of placebo (blue bars)- and RBX (red bars)-treated patients who experienced SMVL in (A) the studies combined (left graph: all patients; top right: only patients with 2 or more years of follow-up; bottom right: only patients with 3 or more years of follow-up) and (B) MBDL (left) and MBCU (right) studies individually. Number of patients shown is the number in each group with postbaseline efficacy evaluations.

TABLE 4. Secondary Analyses

\begin{tabular}{|c|c|c|c|c|c|c|}
\hline \multirow[b]{2}{*}{ Treatment, No. of Patients, ${ }^{*}$ No. of Study Eyes* } & \multicolumn{3}{|c|}{ MBDL } & \multicolumn{3}{|c|}{ MBCU } \\
\hline & $\begin{array}{c}\text { PBO } \\
\text { 360P 696E }\end{array}$ & $\begin{array}{c}\text { RBX } \\
\text { 371P 722E }\end{array}$ & $P$ Value & $\begin{array}{c}\text { PBO } \\
\text { 148P 286E }\end{array}$ & $\begin{array}{c}\text { RBX } \\
\text { 149P 286E }\end{array}$ & $P$ Value \\
\hline Focal photocoagulation, \% patients & 12 & 21 & 0.001 & 24 & 17 & 0.203 \\
\hline DR progression, \% patients & 13 & 12 & 0.475 & 18 & 9 & 0.006 \\
\hline DME COM involvement, \% patients & 20 & 21 & 0.526 & 27 & 27 & 0.971 \\
\hline $\begin{array}{l}\text { VA } 3 \text {-line gain in eyes with VA } 20 / 40 \text { or worse at baseline, } \\
n \text { with event/ } N \text { qualifying points, }(\%)\end{array}$ & $1 / 33(3)$ & $7 / 43(16)$ & 0.071 & $0 / 9(0)$ & $2 / 19(11)$ & $>0.999$ \\
\hline VFQ-25, overall score change from baseline & -1.4 & +0.2 & 0.009 & ND & ND & ND \\
\hline
\end{tabular}

P, patients; E, eyes.

* Numbers represent those with postbaseline measurements. 


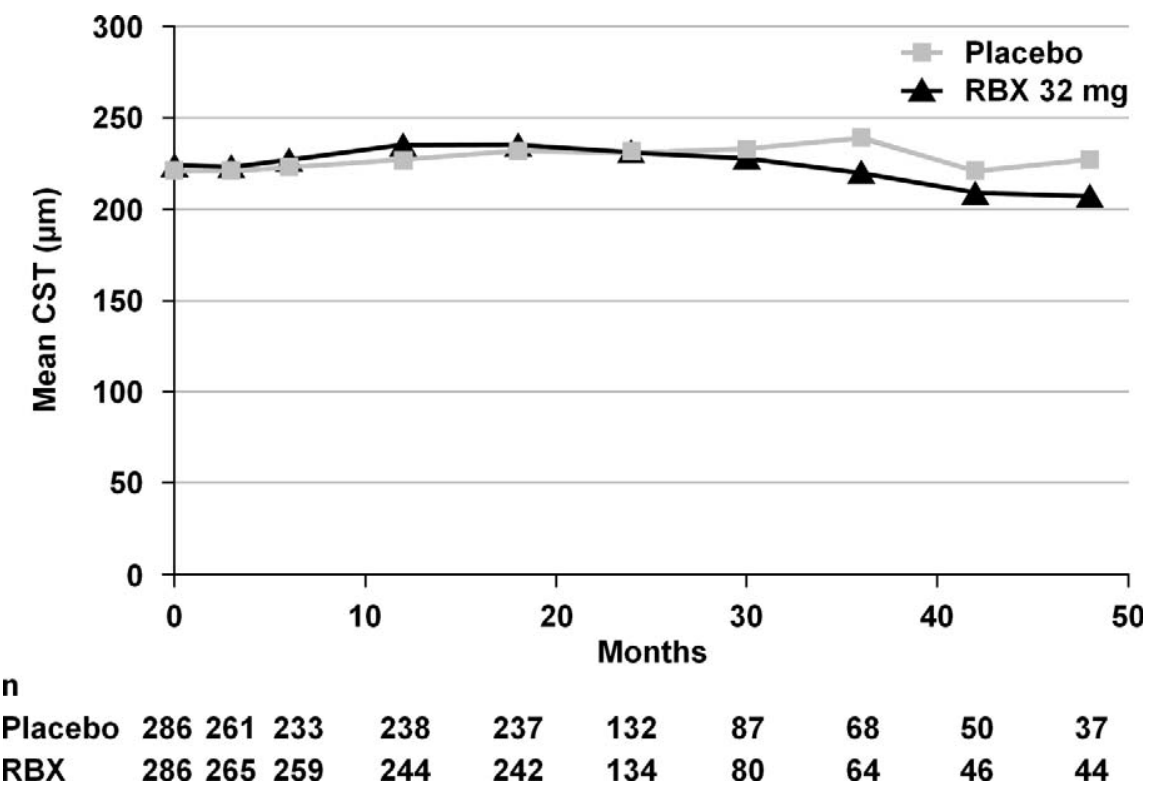

Figure 3. Mean central subfield thickness (CST) for DR study eyes by visit in the MBCU study. The number of patients $(n)$ with available CST data at each time point is shown at the bottom of the figure.

endpoint of both studies. DME severity in Study MBDL was determined through central grading of ETDRS 3-field stereoscopic photographs and was assessed at baseline and every 6 months through the endpoint visit. DME severity in Study MBCU was determined through central grading of ETDRS 7-standard field stereoscopic photographs at baseline to determine study eligibility. Retinal thickness as determined by OCT was also assessed at MBCU baseline and every 6 months through the endpoint visit. Contrast sensitivity was assessed using the Pelli-Robson chart. $^{18}$

At each study visit, patients were queried regarding adverse events. Treatment-emergent adverse events were defined as those that were not present prior to initiation of the study drug, or those that were present prior to initiation of the study drug but worsened either in intensity or in frequency following exposure to the study drug. Serious adverse events were defined as those that resulted in death, hospitalization, life-threatening consequences, severe or permanent disability, or cancer or were significant, in the opinion of the investigator, for any other reason.

Placebo and RBX groups were compared using $\chi^{2}$ or Fisher's exact test for categorical analyses including SMVL, application of FPC, progression of DR, and progression of DME. For the endpoint of SMVL only, a combined study analysis using a Cochran-Mantel-Haenszel $\chi^{2}$ test was performed that included study as a stratifying variable. For continuous variables with multiple visit determinations (including VA, contrast sensitivity, and OCT-determined central macular thickness), a mixed model repeated measures (MMRM) analysis was performed. For the Visual Functioning Questionnaire 25 (VFQ-25), an analysis of covariance (ANCOVA) was performed on baseline to endpoint change in composite score.
Both studies were conducted in accordance with the principles of the Declaration of Helsinki. An institutional review or ethics board approved study conduct at each center, and investigators obtained written informed consent before conducting any study-related procedure.

\section{Results}

Altogether, 1040 patients were randomized, and 822 patients completed their assigned protocol (see Figs. 1A, 1B). Patient characteristics were well matched between the placebo and RBX treatment groups for both studies (Table 2). In addition, patient characteristics across the two studies were very similar in terms of age, sex, origin, diabetes type, duration of diabetes, insulin use, HbA1c, and body mass index (BMI). VA and DR severity were also similar between the two studies. As expected on the basis of differences in eligibility criteria, DME tended to be less severe in MBCU. In both studies, inclusion of fellow eyes that were not required to meet all eligibility requirements broadened the range of DME severity (eyes with no or less than $1 / 6$ optic disc area [DA] of edema approaching 20\% in each study, eyes with CSME approaching $20 \%$ in MBDL, and eyes with CSME within $100 \mu \mathrm{m}$ of the center of the macula approximately $30 \%$ in MBCU) (Table 3). There were no notable differences between treatment groups in either study. Patient disposition was similar across the two studies with overall discontinuation rates in the range of $20 \%$ to $30 \%$ (Figs. 1A, 1B). There were somewhat more discontinuations from the placebo group than from the RBX group in

TABLE 5. Adverse Events

\begin{tabular}{|c|c|c|c|c|}
\hline \multirow[b]{2}{*}{ Treatment, No. of Patients } & \multicolumn{2}{|c|}{ MBDL } & \multicolumn{2}{|c|}{ MBCU } \\
\hline & PBO, $N=360$ & $\mathbf{R B X}, N=371$ & PBO, $N=148$ & $\mathbf{R B X}, N=149$ \\
\hline$\geq 1$ TEAE, $n(\%)$ & $307(85.3)$ & $311(83.8)$ & $118(77.1)$ & $120(80.5)$ \\
\hline$\geq 1 \mathrm{SAE}, n(\%)$ & $81(22.5)$ & $87(23.5)$ & $31(20.3)$ & $36(24.2)$ \\
\hline$\geq 1$ TEAE leading to study discontinuation, $n$ (\%) & $4(1.1)$ & $5(1.3)$ & $4(2.6)$ & $2(1.3)$ \\
\hline Deaths, $n(\%)$ & $9(2.5)$ & $9(2.4)$ & $3(2.0)$ & $2(1.3)$ \\
\hline
\end{tabular}

TEAE, treatment-emergent adverse event; SAE, serious adverse event. 
Study MBCU, with the reason for the majority of the discontinuations reported as "patient decision" (Fig. 1B). Deaths and discontinuations due to adverse events were similar across the two treatment groups and studies (Fig. 1, footnotes).

In the combined analysis across the two studies, SMVL was observed in $4.4 \%$ of 477 patients assigned to placebo versus $2.3 \%$ of 481 assigned to $\operatorname{RBX}(P=0.069$, Fig. $2 \mathrm{~A})$. Additional prospectively specified analyses on the combined SMVL data included only patients with 2 or more years (or 3 or more years) of follow-up to minimize the influence of patients with short follow-up. Similar treatment effects (i.e., RBX versus placebo) were observed in these populations. For the 2-year minimum follow-up group, SMVL was observed in $4.9 \%$ of 385 patients assigned to placebo versus $2.3 \%$ of 397 assigned to RBX $(P=0.045$, Fig. 2A); for the 3-year minimum follow-up group, SMVL was observed in $4.4 \%$ of 318 patients assigned to placebo versus $2.1 \%$ of 334 assigned to $\operatorname{RBX}(P=0.095$, Fig. 2A). SMVL results for each study individually were similar to those observed in the combined analysis (Fig. 2B).

Additional analyses were performed within each study individually and are shown in Table 4.

For the MBDL study MMRM analyses of VA, the estimated mean treatment difference in VA was +0.6 letters (confidence interval $[\mathrm{CI}],-0.01,+1.25, P=0.055$ ) in the direction favoring RBX. For contrast sensitivity, the estimated mean treatment difference in number of letters read correctly was +0.3 letters $(\mathrm{CI},-0.03,+0.68, P=0.072)$ in the direction favoring RBX (data not shown)

For the MBCU study MMRM analyses of VA, the estimated mean treatment difference in VA was +2.3 letters $(\mathrm{CI},+0.3$, $+4.4, P=0.024)$ in the direction favoring RBX. For contrast sensitivity, the estimated mean treatment difference in number of letters read correctly was +0.5 letters $(\mathrm{CI},-0.6,+1.6, P=$ 0.344 ) in the direction favoring RBX (data not shown).

For the MBCU study, MMRM analyses of OCT parameters (center point thickness and center subfield thickness [CST]) did not reveal any consistent differences between treatment groups (data not shown). The mean OCT CST values for DR study eyes at each time point are shown in Figure 3.

An analysis of the safety of RBX on a combined safety database of $>1400$ RBX-treated patients and a similar number of placebo-treated patients has been reported previously. ${ }^{14}$ There were no new safety findings from Studies MBDL and MBCU that changed any of the previous conclusions. Table 5 provides a high-level overview of the percentage of patients who died or who experienced serious adverse events or nonserious treatment-emergent adverse events in each of the two studies.

\section{Discussion}

Although the approximately $50 \%$ reduction of SMVL in the RBX group observed in the overall combined analysis (Fig. 2A), from $4.4 \%(21 / 477)$ in placebo to $2.3 \%(11 / 487)$ in $\operatorname{RBX}(P=0.069)$, did not reach statistical significance, this reduction was consistent with those observed in similar analyses in previous trials of RBX, most notably in the PKC-DRS2, ${ }^{12}$ in which the 9.1\% (31/340) 3-year rate of SMVL in the placebo group was reduced to $5.5 \%(19 / 345)$ with $\mathrm{RBX}$ treatment $(P=0.034)$. In that trial, baseline DR and DME severity were greater and VA was more impaired than in the trials reported here (moderately severe or severe nonproliferative diabetic retinopathy [NPDR] in $91 \%$ vs. $42 \%$ of eyes, DME definitely involving the center of the macula in $31 \%$ vs. $6 \%$, and mean VA 77 vs. 84 letters, respectively). The $9.1 \%$ placebo event rate in that trial compared to the $4.4 \%$ rate reported here was likely due, at least in part, to these differences in disease severity, particularly the 5-fold lower frequency of center-involved DME. Additionally, the lower event rates reported here may be a reflection of increasing use of intravitreal injections of corticosteroids and anti-VEGF agents in recent years. The fact that the use of these treatments was not specifically captured in both trials is a limitation of these studies. A further limitation is the rate of patient discontinuation in both studies $(20 \%-30 \%$ over 2-3 years), although the rate of discontinuation was not higher in the RBX group than in the placebo group.

In previous trials of RBX, the most common probable cause of SMVL was considered to be DME involving the center of the macula $(74 \%$ of eyes had progression to center involvement based upon grading of fundus photographs). ${ }^{2}$ A similar analysis was not performed on the cases of vision loss in Studies MBDL and MBCU, but the similarities in patient populations between these trials and the previous ones make it highly likely that the vision loss in the currently reported trials was primarily due to DME. RBX has been shown to slow progression of DME from earlier stages to involvement of the center, although risk reductions appeared to be smaller than those for prevention of SMVL (approximately 20\% and 40\%, respectively). ${ }^{12,16}$ This difference suggests that the beneficial VA effect of RBX may involve more than reduction of retinal thickening, such as a neuroprotective effect on retinal tissue, and that blocking the PKC $\beta$ pathway alone may not be sufficient to completely inhibit the development/progression of DME. An analysis of the relationship between duration of severe DME and change in VA over a 3-year period also suggests that RBX may provide some protection from the vision-damaging effect of long periods of central retinal thickening. ${ }^{15}$ In contrast to its effects on DME, and unlike the effects of anti-VEGF agents, ${ }^{17} \mathrm{RBX}$ does not appear to influence progression of DR in the populations studied. ${ }^{1,12,16}$ These differences suggest that the beneficial VA effects of RBX may involve mechanisms distinct from those of anti-VEGF agents. Testing of combined treatment of center-involved DME with a selective PKC $\beta$ inhibitor with a favorable safety profile and an intravitreal anti-VEGF agent might be worthy of consideration.

In conclusion, two recently completed randomized clinical trials designed to determine the effects of RBX on slowing the development of DME and its attendant visual loss gave results consistent with those of previous trials, although they failed to meet their primary outcome measure. These trials demonstrated that RBX reduces the relative risk of SMVL from DME by $40 \%$ to $50 \%$, with a lesser benefit on morphologic severity. The greater benefit on functional vision change over structural change may indicate a neuroprotective effect of RBX.

\section{Acknowledgments}

The MBDL and MBCU studies were sponsored by Eli Lilly and Company (Indianapolis, IN). The authors thank the patients who participated in these studies and the physicians and investigative site staff who conducted the studies. We also thank Pamela Anderson of Eli Lilly, who was instrumental in the design and execution of the MBDL study and who provided a valuable review and critique of the manuscript.

\section{References}

1. National Institute of Diabetes and Digestive and Kidney Diseases. National Diabetes Statistics Fact Sheet: General Information and National Estimates on Diabetes in the United States. Bethesda, MD: U.S. Department of Health and Human Services, National Institutes of Health; 2005.

2. Aiello LP, Vignati L, Sheetz MJ, et al. Oral protein kinase C $\beta$ inhibition using ruboxistaurin: efficacy, safety, and causes of vision loss among 813 patients (1,392 eyes) with diabetic 
retinopathy in the protein kinase $C \beta$ inhibitor-diabetic retinopathy study and the protein kinase $C \beta$ inhibitor-diabetic retinopathy study 2. Retina. 2011;31:2084-2094.

3. Brownlee M. Biochemistry and molecular cell biology of diabetic complications. Nature. 2001;414:813-820.

4. Sheetz MJ, King GL. Molecular understanding of hyperglycemia's adverse effects for diabetic complications. JAMA. 2002; 288:2579-2588.

5. Setter SM, Campbell RK, Cahoon CJ. Biochemical pathways for microvascular complications of diabetes mellitus. Ann Pharmacother. 2003;37:1858-1866.

6. Aiello LP, Bursell SE, Clermont A, et al. Vascular endothelial growth factor-induced retinal permeability is mediated by protein kinase $C$ in vivo and suppressed by an orally effective beta-isoform-selective inhibitor. Diabetes. 1997;46:1473-1480.

7. Danis RP, Bingaman DP, Jirousek M, Yang Y. Inhibition of intraocular neovascularization caused by retinal ischemia in pigs by PKC beta inhibition with LY333531. Invest Ophthalmol Vis Sci. 1998;39:171-179.

8. Nonaka A, Kiryu J, Tsujikawa A, et al. PKC beta inhibitor (LY333531) attenuates leukocyte entrapment in retinal microcirculation of diabetic rats. Invest Ophthalmol Vis Sci. 2000; 41:2702-2706

9. Yokota T, Ma RC, Park JY, et al. Role of protein kinase C on the expression of platelet-derived growth factor and endothelin-1 in the retina of diabetic rats and cultured retinal capillary pericytes. Diabetes. 2003;52:838-845.

10. Aiello LP, Clermont A, Arora V, et al. Inhibition of PKC beta by oral administration of ruboxistaurin is well tolerated and ameliorates diabetes-induced retinal hemodynamic abnormalities in patients. Invest Ophthalmol Vis Sci. 2006;47:86-92.

11. The PKC-DRS Study Group. The effect of ruboxistaurin on visual loss in patients with moderately severe to very severe nonproliferative diabetic retinopathy: initial results of the Protein Kinase C beta Inhibitor Diabetic Retinopathy Study (PKC-DRS) multicenter, randomized clinical trial. Diabetes. 2005;54:2188-2197.

12. The PKC-DRS2 Study Group. The effect of ruboxistaurin on visual loss in patients with diabetic retinopathy. Ophthalmology. 2006;113:2221-2230.

13. Girach A, Aiello LP, Milton RC, et al. Sustained moderate visual loss as a predictive end point for visual loss in non-proliferative diabetic retinopathy. Eye. 2009;23:209-214.

14. McGill JB, King GL, Berg PH, et al. Clinical safety of the selective PKC-beta inhibitor, ruboxistaurin. Expert Opin Drug Saf. 2006;5:835-845.

15. Davis MD, Sheetz MJ, Aiello LP, et al., for the PKC-DRS2 Study Group. Effect of ruboxistaurin on the visual acuity decline associated with long-standing diabetic macular edema. Invest Ophthalmol Vis Sci. 2009;50:1-4.

16. PKC-DMES Study Group. Effect of ruboxistaurin in patients with diabetic macular edema. Arch Ophthalmol. 2007;125: 318-324.

17. Nicholson BP, Schachat AP. A review of clinical trials of antiVEGF agents for diabetic retinopathy. Graefes Arch Clin Exp Ophthalmol. 2010;248:915-930.

18. Pelli DG, Robson JG, Wilkins AJ. The design of a new letter chart for measuring contrast sensitivity. Clin Vision Sci. 1988; 2:187-199.

\section{Appendix}

\section{MBDL Study Group}

Australia: Jennifer Arnold, Parramatta, NSW; Mark Gillies, Sydney, NSW; Paul Mitchell, Westmead, NSW; Ian McAllister,
Nedlands, Western Australia; Patrick Phillips, Woodville, South Australia. Brazil: Walter Takahashi, Sao Paulo; Michel Farah, Sao Paulo; Marcos Avila, Goiania; Carlos Moreira, Curitiba. Canada: John Gonder, London, ON; Jean-Daniel Arbour, Montreal, QC; Vladimir Kozousek, Halifax, NS; Iain Begg, Vancouver, BC; Carol Schwartz, Toronto, ON; Heather Lochnan, Ottawa, ON. Denmark: Henrik Lund-Andersen, Glostrup; Toke Bek, Aarhus. France: Pascale Massin, Paris; Michel Weber, Nantes; Jean-Francois Korobelnik, Bordeaux; Saddek Mohand-Said, Paris. Germany: Gabriele Lang, Ulm; Ulrich Mester, Sulzbach; Daniel Pauleikhoff, Munster; Peter Wiedemann, Leipzig. India: Sat Paul Garg, New Delhi; Subhadra Jalali, Hyderabad; Mohan Rema, Chennai; Tarun Sharma, Chennai. Italy: Rosangela Lattanzio, Milano; Paolo Lanzetta, Udine. Mexico: Virgilio Morales-Canton, Mexico City; Jose Guerrero, Mexico City; Arthur Levine, Mexico City. Netherlands: Bettine Polak, Amsterdam; Jose Martinez-Ciriano, Rotterdam. Poland: Miroslawa Polaszewska-Muszynska, Bydgoszcz; Ewa Krzyzagorksa, Poznan; Grazyna Mardarowicz, Lublin; Malgorzata Koziol, Katowice. Portugal: Jose CunhaVaz, Coimbra. Russia: Marina Shestakova, Moscow; Irina Gurieva, Moscow; Natalia Vorokhobina, St. Petersburg; Olga Lantseva, St. Petersburg. Spain: Jose Maria Ruiz Morena, Alicante; Borja Corcostegui, Barcelona; Marta Suarez de Figueroa, Madrid; Maria Isabel Lopez Galvez, Valladolid; Francisco Gomex Ulla, La Coruna. Taiwan: Lai Chi-Chun, Tao-Yuan; Lee Fenq-Lih, Taipei. United Kingdom: Andrew Dick, Bristol; John Forrester, Aberdeen; Paul Dodson, Birmingham; Jiten Vora, Liverpool; Philip Hykin, London. United States of America: Thomas Chandler, Austin, TX; Nauman Chaudhry, Guilford, CT; Gary Fish, Dallas, TX; Robert Foster, Cincinnati, OH; Mark Chiu, Albuquerque, NM; Ronald Gentile, New York City, NY; Jon Gieser, Wheaton, IL; Roy Goodart, Salt Lake City, UT; Craig Greven, Winston-Salem, NC; Dean Hainsworth, Columbia, MO; Bruce Keyser, New Brunswick, NJ; Robert Leonard, Oklahoma City, OK; Jeffrey Marx, Peabody, MA; Scott Richards, Ogden, UT; George Sharuk, Boston, MA; Lawrence Singerman, Beachwood, OH; Keye Wong, Sarasota, FL; Frank Garber, Grand Rapids, MI; Prema Abraham, Rapid City, SD; Andrew Antoszyk, Charlotte, NC; David Brown, Houston, TX; Stuart Burgess, Sunrise, FL; Clement Chan, Palm Springs, CA; Doug Dehning, Independence, MO; Craig Leong, Walnut Creek, CA; Daniel Finkelstein, Baltimore, MD; Stephen Russell, Iowa City, IA; Steven Madreperla, Teaneck, NJ; John Puglisi, North Miami, FL; David Quillen, Hershey, PA; Denis Marcus, Augusta, GA; Jeffrey Levenson, Jacksonville, FL; Peter Kaiser, Cleveland, OH; Bernard Doft, Pittsburgh, PA; Wayne Solley, Arlington, TX; Glenn Stoller, Rockville Center, NY; Paul Tornambe, Poway, CA; Max Johnson, Fargo, ND; Kenneth Sall, Artesia, CA; Aaron Affleck, Idaho Falls, ID.

\section{MBCU Study Group}

Canada: Fareed Ali, Mississauga, ON; John Gonder, London, ON; Vladimir Kozousek, Halifax, NS. Denmark: Henrik LundAndersen, Glostrup; Toke Bek, Aarhus. France: Pascale Massin, Paris; Saddek Mohan-Said, Paris; Michel Weber, Nantes. Germany: Gabriele Lang, Ulm; Daniel Pauleikhoff, Munster; Nicole Eter, Bonn; Frank Holz, Bonn; Peter Wiedemann, Leipzig. Lithuania: Ingrida Januleviciene, Kaunas. Mexico: Virgilio Morales, Mexico City; Jorhe Fromow, Mexico City; Nathan Grinberg, Mexico City; Patricio Rodriguez, Monterrey; Cynthia Espinosa Martinez, San Luis Potosi. Portugal: Jose Cunha-Vaz, Coimbra; Fernando Falcao-Reis, Porto. Romania: Simona Barsan, Bucuresti; Mary Liliana Voinea, Bucuresti. Spain: Jose Maria Ruiz Moreno, Alicante; Isabel Lopez Galvez, Valladolid; Borja Corcostegi Guraya, Barcelona; Javier Montero Moreno, Valladolid. United Kingdom: Phillip Hykin, London; 
Sobha Sivaprasad, London. United States of America: Rajiv Anand, Dallas, TX; Bernard Doft, Pittsburgh, PA; Daniel Finkelstein, Baltimore, MD; Paula Ko, Newark, DE; Jeffrey Levenson, Jacksonville, FL; Peter Kaiser, Cleveland, $\mathrm{OH}$; Kenneth Sall, Artesia, CA; David Miller, Lakewood, OH; George
Sharuk, Boston, MA; David Brown, Houston, TX; Raj Maturi, Indianapolis, IN; Naomi Falk, Slingerlands, NY; Alan Gordon, Phoenix, AZ; Dennis Marcus, Augusta, GA; Heidi Becker, San Antonio, TX; Cameron Stone, Asheville, NC; David Miller, Beachwood, $\mathrm{OH}$. 